\title{
The least-squares ambiguity decorrelation adjustment: a method for fast GPS integer ambiguity estimation
}

\author{
P. J. G. Teunissen \\ Delft Geodetic Computing Centre (LGR), Faculty of Geodetic Engineering, Delft University of Technology, Thijsseweg 11, 2629 JA Delft, \\ The Netherlands
}

Received 7 July 1994; Accepted 14 November 1994

\begin{abstract}
The GPS double difference carrier phase measurements are ambiguous by an unknown integer number of cycles. High precision relative GPS positioning based on short observational timespan data, is possible, when reliable estimates of the integer double difference ambiguities can be determined in an efficient manner. In this contribution a new method is introduced that enables very fast integer leastsquares estimation of the ambiguities. The method makes use of an ambiguity transformation that allows one to reformulate the original ambiguity estimation problem as a new problem that is much easier to solve. The transformation aims at decorrelating the least-squares ambiguities and is based on an integer approximation of the conditional leastsquares transformation. This least-squares ambiguity decorrelation approach, flattens the typical discontinuity in the GPS-spectrum of ambiguity conditional variances and returns new ambiguities that show a dramatic improvement in correlation and precision. As a result, the search for the transformed integer least-squares ambiguities can be performed in a highly efficient manner.
\end{abstract}

\section{Contents}

1. Introduction

2. The GPS Ambiguity Estimation Problem

2.1 The Carrier-Phase Observation Equation

2.2 Integer Least-Squares Estimation

2.3 Sequential Conditional Least-Squares Estimation

2.4 Search for the Integer Least-Squares Ambiguities

2.5 The GPS Spectrum of Ambiguity Conditional Variances

3. The Reparametrized Ambiguity Estimation Problem

3.1 The Class of Admissible Ambiguity Transformations

3.2 A 2D-Decorrelating Ambiguity Transformation

\subsection{Flattening the Spectrum of Ambiguity} Conditional Variances

3.4 On the Spectrum and the Gain in Baseline Precision

4. Summary and Concluding Remarks

5. References

\section{Introduction}

High precision relative GPS positioning is based on the very precise carrier phase measurements. A prerequisite for obtaining high precision relative positioning results, is that the double-difference carrier phase ambiguities become sufficiently separable from the baseline coordinates. Different approaches are in use and have been proposed to ensure a sufficient separability between these two group of parameters [1-8]. In particular, the approaches that explicitly aim at resolving the integer-values of the double-difference ambiguities have been very successful. Once the integer ambiguities are fixed, the carrier phase measurements will start to act as if they were high-precision pseudorange measurements, thus allowing for a baseline solution with a comparable high precision. However, the fixing of the integer ambiguities is a non-trivial problem, in particular if one aims at numerical efficiency. This topic has therefore been a rich source of GPS-research over the last decade or so. Starting from rather simple but timeconsuming integer rounding schemes, the methods have evolved into complex and effective search algorithms [9-17]. Nevertheless, at present times, it is still expedient to seek ways for improving the efficiency of the various search methods. This is in particular true for real-time applications of GPS. But to a certain extent, this is also true for some typical static applications of GPS. If we are namely really able to significantly reduce the computational effort for estimating the integer ambiguities, it may also become worthwhile to tackle problems that have dimensions higher than the one's considered sofar. For instance, it may become much easier then to simultaneously 
estimate all integer double-difference ambiguities when adjusting a GPS network [18].

In this contribution a new method will be presented for the integer least-squares estimation of the double-difference carrier phase ambiguities. The method consists of two steps. In the first step, an ambiguity transformation $Z^{*}$ is constructed that allows one to reparametrize the original double-difference ambiguities, such that new ambiguities are obtained, having certain desirable properties. Hence, the original integer ambiguity vector $a$, its real-valued leastsquares estimate $\hat{a}$ and corresponding variance-covariance matrix $Q_{\hat{a}}$, are transformed as

$$
z=Z^{*} a, \hat{z}=Z^{*} \hat{a}, Q_{\hat{z}}=Z^{*} Q_{\hat{a}} Z .
$$

The ambiguity transformation $Z^{*}$ is required to be integer and volume preserving [19]. The kernelletter $z$ is used to denote the transformed ambiguities. The idea of transforming the double-difference ambiguities is of course not completely new. Certain linear combinations of the GPS-observables already play a prominent role in the problem of ambiguity fixing. In particular "wide-laning" techniques have proven to be very successful. Well-known examples are the narrowlane, the wide-lane and extra wide-lane combinations [20-22]. But also other wide-lane combinations have been studied [23]. At present however, the various integer linear combinations that are considered, are restricted to the singlechannel dual-frequency case. They therefore require the use of dual-frequency carrier phase data. Moreover, since they enact at the level of a single channel only, they do not allow one to take care of the receiver-satellite geometry. The above ambiguity transformation $Z^{*}$ however, does not require the use of dual-frequency carrier phase data per se and also allows one to take care of the slowly changing receiversatellite geometry, a geometry which is so emphatically present in the ambiguity variance-covariance matrix $Q_{\hat{a}}$.

Once the transformed ambiguities have been obtained, the actual search for the integer least-squares ambiguities is performed in the second step. The search is based on a sequential conditional least-squares adjustment of $\hat{z}$ and it derives its efficiency from the properties of the transformed ambiguities. In terms of the conditional least-squares ambiguity estimates, the transformed ambiguity searchspace is described by the inequality

$$
\sum_{i=1}^{n}\left(\hat{z}_{i \mid l}-z_{i}\right)^{2} / \sigma_{\hat{z}(|| I, i \mid)} \leq \chi^{2}
$$

in which $\hat{z}_{i \mid I}$ denotes the least-squares estimate of the $i$ th ambiguity conditioned on the first $(i-1)$ number of ambiguities and $\sigma_{\hat{z}(i|,|, \mid])}$ denotes its variance. $\chi^{2}$ is an appropriately chosen positive constant that ensures that the ambiguity searchspace indeed contains the sought for integer least-squares ambiguities. It is the sum-of-squares structure in the above inequality that allows one to formulate bounds for the individual ambiguities, thus enabling that a search for the transformed integer least-squares ambiguity $\check{z}$ can be performed. Although the recovery of a sum-of-squares structure is not new in itself, there are important differences in how it is interpreted and used for the search [13],[16,17],[24]. Once the integer estimate $\check{z}$ has been determined, the corresponding baseline solution $\check{b}$ can be recovered as

$$
\check{b}=\hat{b}-Q_{\hat{b} \hat{z}} Q_{\hat{z}}^{-1}(\hat{z}-\check{z}),
$$

in which $\hat{b}$ denotes the non-fixed least-squares baseline solution.

The basic idea that lies at the root of the method - both in the construction of $Z^{*}$, as in the formulation of the search is, that integer least-squares ambiguity estimation becomes trivial once all the least-squares ambiguities are fully decorrelated. Their confidence ellipsoid would then be aligned with the grid axes and the sought for integer leastsquares ambiguities would then simply follow from a rounding of the real-valued least-squares estimates to their nearest integer. The ambiguities would be fully decorrelated when the conditional least-squares estimates are identical to their unconditional counterparts, i.e. when $\hat{a}_{i \mid t} \equiv \hat{a}_{i}$ holds true for all $i$. In case of GPS however, the least-squares ambiguities are usually highly correlated and their confidence ellipsoid is usually extremely elongated. This is particularly true in case of short observational timespans and in the absence of precise P-code data. As a consequence of the intrinsic structure of the ambiguity variance-covariance matrix, the spectrum of ambiguity conditional variances, $\sigma_{\hat{a}(i|I, i|)} i=1, \ldots, n$, generally shows a large discontinuity when passing from the third to the fourth conditional variance. But this implies, when the above inequality is used in the original parametrization, that the search would suffer from the fact that the bounds for the first three ambiguities are rather loose, whereas the remaining bounds are extremely tight. The essence of the method is therefore to aim at constructing a decorrelating ambiguity transformation $Z^{*}$, that removes the discontinuity from the spectrum. In two dimensions this is achieved by basing the ambiguity transformation on an integer approximation of the fully decorrelating conditional least-squares transformation. Transformations of this type are also known as Gauss-transformations and in the non-integer case they are considered to be the basic tools for zeroing 
entries in matrices [25]. The $n$-dimensional case is tackled through a repeated use of the two-dimensional decorrelating ambiguity transformation. But instead of applying it to the unconditional ambiguities, it is now applied to pairs of conditional least-squares ambiguities. This approach has been motivated by the presence of the typical discontinuity in the GPS spectrum of ambiguity conditional variances and is based on ideas from [26]. The success of our method is largely due to the presence of this discontinuity and it stipulates the relevance of satellite redundancy and the use of dual-frequency data. Once the spectrum of conditional variances has been flattened, less correlated and very precise ambiguities $\hat{z}_{i}$ are returned, thus allowing that the search for the transformed integer least-squares ambiguities can indeed be performed in a highly efficient manner.

In order to properly judge the significance of the present contribution, it is important that one distinguishes between the following two problems of GPS-ambiguity fixing:

1. The ambiguity estimation problem, and

2. The ambiguity validation problem.

The present contribution only addresses the first problem and not the second. The second problem, which depends on the outcome of the first, is concerned with the validation of the estimated integer ambiguities. Although the procedures for validating the estimated ambiguities which are currently in use in practice, appear to work satisfactory, it is the author's opinion that there is still some room for improving the theoretical basis of these validation procedures. For a proper statistical evaluation of both the estimated ambiguities as well as the corresponding baseline solution, it would be very helpful indeed if one has at one's disposal the probability densities of the corresponding integer estimators. This however, is a non-trivial problem, but reference is made to the discussions in [13], [27-29]. Despite the importance of proper validation procedures, the present contribution is only concerned with the integer ambiguity estimation problem. There is therefore no harm in stressing, that our method, efficient as it may be, might still come up with the wrong integer ambiguities if the data are contaminated with unmodelled effects.

\section{The GPS Ambiguity Estimation Problem}

In this chapter the integer least-squares ambiguity estimation problem is formulated and discussed. It shows how the search can be based on a sequential conditional least-squares adjustment of the ambiguities and it explains why in case of GPS such a search, when performed for the original ambiguities, suffers from being inefficient. As such, chapter two identifies the dilemma with which one is confronted and shows along which lines solutions can be sought. In section 2.1, first the basic observation equation of the carrier phase observable is given. We restrict our attention to carrier phase data to accentuate that our method is in principle independent of the use of code data or even the use of dual-frequency data. But the method can easily accommodate code data as well, although with Anti-Spoofing turned on, very precise code data will probably no longer be available. The doubledifference version of the carrier phase observation equation forms the basis of the model that will be used for the integer least-squares ambiguity estimation. The concept of integer least-squares estimation is discussed in section 2.2. In section 2.3 a sequential conditional least-squares estimation of the ambiguities is performed in order to recover a sum-ofsquares representation for the ambiguity search space. The sum-of-squares structure is used to formulate sharp bounds for the individual ambiguities. The search procedure based on these bounds, is discussed in section 2.4. In section 2.5 the spectrum of ambiguity conditional variances is studied. It is shown, when short observational timespan carrier phase data are used, that the special structure of the variancecovariance matrix of the ambiguities usually results in a spectrum having a large discontinuity. As a consequence, the search for the original ambiguities suffers from the potential problem of halting.

\subsection{The Carrier-Phase Observation Equation}

The GPS observables are ( $P$ or $C / A)$ code-derived pseudorange measurements and carrier phase measurements. These data can be available on both of the two frequencies $L_{1}$ and $L_{2}$. In the following we will restrict our attention to the carrier phase measurement $\Phi$. The carrier phase measurement can be represented as

$$
\Phi=\|r-R\|+c(d t-d T)+\lambda N+\varepsilon,
$$

where:

$r \quad: \quad$ is the unknown receiver antenna position vector at signal reception time,

$R \quad$ : is the given satellite antenna position vector at signal transmission time,

$c:$ : is the speed of light in vacuum,

$c d t \quad:$ is the receiver clock range offset,

$c d T:$ is the satellite clock range offset,

$\lambda \quad:$ is the carrier wavelength of the signal $\left(L_{1}\right.$ or $\left.L_{2}\right)$, 
and

$N \quad$ : is the carrier phase ambiguity $\left(L_{1}\right.$ or $\left.L_{2}\right)$.

The term $\varepsilon$ represents carrier phase measurement noise and biases such as satellite ephemeris errors, tropospheric and ionospheric delays, and ranging errors caused by multipath. In the processing of phase data it is not uncommon to difference the carrier phase measurements between satellites and between receivers to eliminate the satellite and receiver clock offsets. This gives the double difference carrier phase observation equation

$$
D D \Phi=D D\|r-R\|+\lambda D D N+D D \varepsilon,
$$

where $D D$ stands for the double-difference operator. In this equation the ambiguity-term $D D N$ is known to be integervalued. For reasons of simplicity we will restrict ourselves in the following to the two-receiver situation. This however, has no effect on the general applicability of the proposed method. The method is namely independent of the number of receivers used, and is therefore also applicable in situations where more than two receivers are used. This is for instance the case when the GPS-data are simultaneously adjusted for in a network-mode.

Linearization of the observation equations with respect to the unknown parameters, and a collection of these linearized equations into a linear system of equations, gives

$$
y=A a+B b+e,
$$

where:

$y \quad: \quad$ is the vector of observed minus computed double difference carrier phase measurements,

$a \quad:$ is the vector of unknown integer double difference ambiguities,

$b \quad: \quad$ is the vector that contains the increments of the unknown baseline components,

$A, B \quad$ : are the design matrices for ambiguity terms and baseline components, and

$e \quad: \quad$ is the vector of unmodelled errors.

This system of observation equations is taken as point of departure for computing estimates of the unknown parameters $a$ and $b$. Our estimation criterion will be based on the principle of least-squares. From a statistical viewpoint this choice is motivated by the fact that, in the absence of modelling errors, properly weighted linear least-squares estimators are identical to unbiased minimum variance estimators. Furthermore, these estimators are also maximum likelihood estimators if the assumption of normality holds for the phase observables. In the following we will assume that the bias-terms in $e$ are either corrected for or sufficiently small to be neglected.

\subsection{Integer Least-Squares Estimation}

The least-squares criterion for solving the linear(ized) system of observation equations (6) reads

$$
\min _{a, b}\|y-A a-B b\|_{Q_{y}}^{2} \text { with } a \in Z^{n}, b \in R^{3} \text {, }
$$

where \|\|$_{Q_{y}}^{2}=(.)^{*} Q_{y}^{-1}(),. Q_{y}$ is the variance-covariance matrix of the double-difference carrier phase observables, $Z^{n}$ is the $n$-dimensional space of integers and $R^{3}$ is the 3 dimensional space of reals. The minimizers of (7) will be denoted as respectively $\breve{a} \in Z^{n}$ and $\check{b} \in R^{3}$. Note that (7) is a constrained least-squares problem. This is due to the presence of the integer-constraint $a \in Z^{n}$. The minimization problem (7) will therefore be referred to as an integer leastsquares problem.

The quadratic objective function of the above integer leastsquares problem can be decomposed into a sum of three squares,

$$
\|y-A a-B b\|_{Q_{y}}^{2}=\|\hat{e}\|_{Q_{y}}^{2}+\|\hat{b} \mid a-b\|_{Q_{\hat{k} \mid a}}^{2}+\|\hat{a}-a\|_{Q_{a}}^{2},
$$

where: $\hat{a}$ is the real-valued, unconstrained least-squares ambiguity vector, having $Q_{\hat{a}}$ as variance-covariance matrix $; \hat{b} \mid a$ is the conditional least-squares baseline vector, conditioned on $a$, having $Q_{\hat{b} \mid a}$ as variance-covariance matrix; and $\hat{e}$ is the unconstrained least-squares residual vector. From the above decomposition follows, that the last two squares vanish identically, if the objective function (8) would be minimized as function of $a \in R^{n}$ and $b \in R^{3}$. Hence, the minimizers would then be given by $\hat{a} \in R^{n}$ and $\hat{b} \in R^{3}$, and the minimum of the objective function would be given by the squared norm of the least-squares residual vector $\hat{e}$. In our case however, the objective function needs to be minimized as function of $a \in Z^{n}$ and $b \in R^{3}$. In that case, only the second square vanishes identically and the minimizers are given by $\check{a} \in Z^{n}$ and $\check{b}=\hat{b} \mid \check{a} \in R^{3}$. The corresponding minimum of the objective function is then given by $\|\hat{e}\|_{Q_{y}}^{2}+\|\hat{a}-\breve{a}\|_{Q_{a}}^{2}$.

The above shows that the integer least-squares problem (7) may be solved in two steps. The first step consists then of solving (7) with $Z^{n}$ replaced by $R^{n}$. Hence, in the first step the integer-constraint is removed, reducing the problem to an ordinary unconstrained least-squares problem. As a result of this first step, real-valued estimates for both the ambiguities, 
$\hat{a}$, and the baseline components, $\hat{b}$, are obtained, together with their corresponding variance-covariance matrices:

$$
\left(\begin{array}{l}
\hat{a} \\
\hat{b}
\end{array}\right),\left(\begin{array}{ll}
Q_{\hat{a}} & Q_{\hat{a} \hat{b}} \\
Q_{\hat{b} \hat{a}} & Q_{\hat{b}}
\end{array}\right) .
$$

This result forms then the input for the second step. In the second step one first solves for the vector of integer leastsquares estimates of the ambiguities, $\breve{a}$. It follows from solving the integer least-squares problem

$$
\text { min. }(\hat{a}-a) Q_{\hat{a}}^{-1}(\hat{a}-a) \text { with } a \in Z^{n} \text {. }
$$

Once the integer least-squares ambiguity vector $\check{a} \in Z^{n}$ has been obtained, the residual $(\hat{a}-\check{a})$ is used to adjust the unconstrained baseline solution $\hat{b}$, to get $\check{b}=\hat{b} \mid \breve{a}$. As a result, the final baseline solution is obtained as

$$
\check{b}=\hat{b} \mid \check{a}=\hat{b}-Q_{\hat{b} \hat{a}} Q_{\hat{a}}^{-1}(\hat{a}-\check{a}) .
$$

The above step-wise approach agrees conceptually with the procedure that is usually followed in practice, when ambiguity-fixing is included in the baseline computations. The estimates $\hat{a}$ and $\hat{b}$ are sometimes referred to as the "float-solution", and the estimates $\check{a}$ and $\check{b}$, being the solution of (7), are then referred to as the "fixed-solution".

Note that the minimization problem (10) may not have a unique solution. Although it is very unlikely that it has more than one solution, it is possible in principle that (10) has up to $2^{n}$ different integer minimizers. Still however, we will assume in the present sequel that (10) has one and only one solution. Our motivation for this assumption is based on the fact, that a reliable fixing of the ambiguities will not be considered feasible whenever the solution of (10) is nonunique.

The variance-covariance matrix of $\hat{b} \mid a$ is given as $Q_{\hat{b} \mid a}=Q_{\hat{b}}-Q_{\hat{b} \hat{a}} Q_{\hat{a}}^{-1} Q_{\hat{a} \hat{b}}$. In practice, this variance-covariance matrix is usually used to describe the precision of the final baseline solution $\check{b}$ (approaches may differ, however, in the way this matrix is scaled). Here however, some remarks of caution are in order [27]. The structure of (11) shows, that $\check{b}$ can be interpreted as a conditional least-squares estimate. That is, starting from the result of the first step, (9), $\check{b}$ can be interpreted as the least-squares estimate of $b$, conditioned on the integer ambiguity-vector $\breve{a}$. And consistent with this interpretation, the precision of $\breve{b}$ may indeed be described by the variance-covariance matrix $Q_{\hat{b} \mid a}$. When doing so, one should recognize however, that $Q_{\hat{b} \mid a}$ is interpreted as a conditional variance-covariance matrix. Hence, in the light of predicting empirical outcomes of future experiments, the following meaning should be given to the formal variancecovariance matrix $Q_{\hat{b} \mid a}$. When the measurement experiment is repeated a sufficient number of times under similar circumstances, the matrix $Q_{\hat{b} \mid q}$ describes the spread one can expect in the various baseline solutions, when all baseline solutions are based on the same value for $a$. But this shows, since in practice each baseline determination will have its own integer estimate for the ambiguity vector $a \in Z^{n}$, that strictly speaking, $Q_{\hat{b} \mid a}$ does not describe what it should describe. In other words, $Q_{\hat{b} \mid a}$ is the variance-covariance matrix of $\hat{b} \mid a$, but not necessarily that of $\check{b}=\hat{b} \mid \check{a}$. The fact that the least-squares estimator of the ambiguity vector is integer, does not imply that it is nonstochastic. What is needed therefore, is the unconditional variance-covariance matrix of $\breve{b}$. Hence, in order to obtain the theoretically correct variance-covariance matrix of $\check{b}$, the stochasticity of the integer estimator of the ambiguity vector should be taken into account when applying the error propagation law to (11). This is a nontrivial problem (the probability density function of $\check{a}$ is of the discrete type) and one that has not yet been solved satisfactorily from a theoretical point of view. Fortunately, the practical relevance of this problem may be minor, in particular when a sound validation procedure has been used for the validation of $\breve{a}$. One of the features of a proper validation procedure should namely be to verify whether or not sufficient probability mass is located at a single gridpoint of $Z^{n}$. And when this can be assured to a sufficient degree, the influence of the stochasticity of $\check{a}$ on $\check{b}$ will be small and matrix $Q_{\hat{b} \mid a}$ can be taken as a sufficient realistic measure for the precision of $\check{b}$.

It will be clear that $Q_{\hat{b} \mid a}<Q_{\hat{b}}$. When short observational timespans are used, we in fact have $Q_{\hat{b} \mid a} \ll<Q_{\hat{b}}$. This can be explained as follows. Since GPS satellites are in very high altitude orbits, their relative positions with respect to the receiver change slowly, which implies in case of short observational timespans, that the ambiguities - when treated as being real-valued - become very poorly separable from the baseline coordinates. As a result, the precision with which the baseline can be estimated will be rather poor. However, when one explicitly aims at resolving for the integer-values of the ambiguities and assumes that their discrete probability density function is sufficiently peaked, the high-precision carrier-phase observables will start to act as if they were high-precision pseudo-range observables. As a result, the baseline coordinates become estimable with a comparable high precision and $Q_{\grave{b}} \cong Q_{\hat{b} \mid a} \ll<Q_{\hat{b}}$ holds true. The sole purpose of "ambiguity-fixing" is thus, to be able, via the 
inclusion of the integer-constraint $a \in Z^{n}$, to obtain a drastic improvement in the precision of the baseline solution. In this way, long observational timespans can be avoided, which otherwise would have been needed if the ambiguities were treated as being real-valued.

In the remaining of the sequel we will not be concerned directly with the baseline solution. Instead we will concentrate our attention on solving the ambiguity integer least-squares problem (10). And it is with the minimization of this constrained objective function, that the intricacy of the integer ambiguity estimation problem manifests itself.

\subsection{Sequential Conditional Least-Squares Estimation}

Due to the presence of the integer-constraint $a \in Z^{n}$, there are unfortunately in general no standard techniques available for solving (10) as they are available for solving ordinary least-squares problems. As a consequence one has to resort to methods that in one way or another make use of a discrete search strategy for finding the integer minimizer of (10). However, before we start thinking of setting up such a search strategy, it helps if we ask ourselves the question what the structure of (10) must be in order to be able to apply the simplest of all integer estimation methods. Clearly, the simplest integer estimation method is "rounding to the nearest integer". And when applied to (10), this method will give the correct answer provided that the ambiguity variancecovariance matrix $Q_{\hat{a}}$ is diagonal, i.e. when all least-squares ambiguities are fully decorrelated. A diagonal $Q_{\hat{a}}$ implies namely that (10) reduces to the minimization of a sum of independent squares,

$$
\underset{a_{1} \ldots a_{1} \in Z}{\operatorname{minimize}} \sum_{i=1}^{n}\left(\hat{a}_{i}-a_{i}\right)^{2} / \sigma_{\hat{a}(i, i)},
$$

where $\sigma_{\hat{a}(i, i)}$ denotes the variance of the $i$ th least-squares ambiguity. Hence, in that case we can work with $n$ separate scalar integer least-squares problems. And the integer minimizers of each of these individual squares are then simply given by the integers nearest to $\hat{a}_{i}$. The conclusion reads therefore, that the ambiguity integer least-squares problem becomes trivial, when all least-squares ambiguities are fully decorrelated.

Unfortunately, in reality, the least-squares ambiguities are usually highly correlated and the variance-covariance matrix $Q_{\hat{a}}$ is far from being diagonal. Still however, it is possible to recover a sum-of-squares structure of the objective function if we diagonalize $Q_{\hat{a}}$. Not every diagonalization works however. What is needed in addition, is, that the diagonalization realizes, like in (12), that the individual ambiguities can be assigned to the individual squares in the total sum-of-squares. This for instance, rules out a diagonalization based on an eigenvalue decomposition of the ambiguity variance-covariance matrix. In the same spirit of decomposition (8), we will therefore apply a conditional least-squares decomposition to the ambiguities. And this will be done on an ambiguity-by-ambiguity basis. Hence, a sequential conditional least-squares estimation will be applied. This implies that the first least-squares ambiguity $\hat{a}_{1}$ remains unchanged. The second least-squares ambiguity however, is replaced by its least-squares estimate, conditioned on the first ambiguity $a_{1}$. This gives $\hat{a}_{2 \mid 1}=\hat{a}_{2}-$ $\sigma_{\hat{a}(2,1)} \sigma_{\hat{a}(1,1)}^{-1}\left(\hat{a}_{1}-a_{1}\right)$. Note that $\hat{a}_{2 \mid 1}$ is uncorrelated with $a_{1}$. The third least-squares ambiguity is replaced by its leastsquares estimate conditioned on the first two ambiguities $a_{1}$ and $a_{2}$. This gives $\hat{a}_{3 \mid 2,1}=\hat{a}_{3}-\sigma_{\hat{a}(3,1)} \sigma_{\hat{a}(1,1)}^{-1}\left(\hat{a}_{1}-a_{1}\right)-$ $\sigma_{\hat{a}(3,2 \mid 1)} \sigma_{\hat{a}(2|1,2| 1)}^{-1}\left(\hat{a}_{2 \mid 1}-a_{2}\right)$. Note that $\hat{a}_{3 \mid 2,1}$ is uncorrelated with both $\hat{a}_{2 \mid 1}$ and $\hat{a}_{1}$. By continuing in this way, we obtain for the ith step, using the shorthand notation $\hat{a}_{j \mid J}$ for $\hat{a}_{j \mid(j-1) \ldots 1}$,

$$
\hat{a}_{i \mid I}=\hat{a}_{i}-\sum_{j=1}^{i-1} \sigma_{\hat{a}(i, j \mid J)} \sigma_{\hat{a}(j|J, j| J)}^{-1}\left(\hat{a}_{j \mid J}-a_{j}\right) .
$$

And $\hat{a}_{i \mid I}$ is uncorrelated with all $\hat{a}_{j \mid J}$ for $j=1, \ldots,(i-1)$. It now follows from (13) that the ambiguity difference $\left(\hat{a}_{i}-a_{i}\right)$ can be written in terms of the differences $\left(\hat{a}_{j \mid J}-a_{j}\right), j=1, \ldots, i$, as $\left(\hat{a}_{i}-a_{i}\right)=\left(\hat{a}_{i \mid I}-a_{i}\right)+\sum_{j=1}^{i-1} \sigma_{\hat{a}(i, j \mid J)} \sigma_{\hat{a}\left(j\left|J_{j}\right| J\right.}^{-1}\left(\hat{a}_{j \mid J}-a_{j}\right)$. Hence, when this is written out in vector-matrix form, using the notation $\hat{d}=\left(\hat{a}_{1}, \hat{a}_{2 \mid 1}, \ldots, \hat{a}_{n \mid N}\right)^{*}$, and the error propagation law is applied, it follows, because of the fact that the conditional least-squares ambiguities are mutually uncorrelated, that

$$
(\hat{a}-a)=L(\hat{d}-a) \text { and } Q_{\hat{a}}=L D L^{*},
$$

where: $\quad D=\operatorname{diag} .\left(\ldots, \sigma_{\hat{a}(i|,|, \mid l)}, \ldots\right)$ and $(L)_{i j}=0$ for $1 \leq i<j \leq n$, and $(L)_{i j}=1$ for $i=j$, and $(L)_{i j}=\sigma_{\hat{a}(i, j \mid J)} \sigma_{\hat{a}(j|J, j| J)}^{-1}$ for $1 \leq j<i \leq n$. The above matrix decomposition is well-known and is usually referred to as the $L D L^{*}$-decomposition [25]. With our "re-discovery" of the $L D L^{*}$-decomposition, we now can give a clear statistical interpretation to each of the entries of the lower triangular matrix $L$ and to each of the entries of the diagonal matrix $D$. This interpretation will also be of help for the construction of the ambiguity transformation in the next chapter. Substitution of (14) into the objective function of (10) gives the desired sum-of-squares structure, and allows us to rewrite the integer least-squares problem as

$$
\underset{a_{1} \cdots a_{n} \in Z}{\operatorname{minimize}} \sum_{i=1}^{n}\left(\hat{a}_{i \mid I}-a_{i}\right)^{2} / \sigma_{\hat{a}(i|I, i| n)} .
$$

Note that the sum-of-squares of (15) reduces to that of (12), when all least-squares ambiguities would be fully decorrelated. Also note, that in case of (15), a simple 
"rounding to the nearest integer" will now not necessarily give the correct integer minimizer to (10). This is due to the fact that $\hat{a}_{i \mid l}$ depends on $a_{j}$ for $j=1, \ldots,(i-1)$. But, the sum-ofsquares structure of (15) still allows one to set up sharp bounds for the individual ambiguities. This is shown in the next section.

\subsection{Search for the Integer Least-Squares Ambiguities}

In order to solve (15) we will first restrict the solution space by replacing the space of integers, $Z^{n}$, by a smaller subset that can be enumerated. The idea is to use the objective function of (15) for introducing an ellipsoidal region in $R^{n}$, on the basis of which the search can be performed. This ellipsoidal ambiguity search space is determined by

$$
\sum_{i=1}^{n}\left(\hat{a}_{i \mid l}-a_{i}\right)^{2} / \sigma_{\hat{a}(i|I, i| I)} \leq \chi^{2} .
$$

This ellipsoidal region is centred at $\hat{a} \in R^{n}$, its orientation and elongation are governed by the ambiguity variancecovariance matrix $Q_{\hat{a}}$, and its size can be controlled through the selection of the positive constant $\chi^{2}$. It will be assumed that the positive constant $\chi^{2}$ has been chosen such that the region at least contains the sought for integer minimizer of (15) [24].

In order to discuss our search for the integer least-squares ambiguities, we will first consider the two-dimensional case. For $n=2$, the inequality (16) reduces to

$$
\left(\hat{a}_{1}-a_{1}\right)^{2} / \sigma_{\hat{a}(1,1)}+\left(\hat{a}_{2 \mid 1}-a_{2}\right)^{2} / \sigma_{\hat{a}(2|1,2| 1)} \leq \chi^{2} .
$$

This ellipsoidal region is shown in figure 1. Also shown in the figure are the rectangular box that encloses the ellipse and the line passing through the centre of the ellipse, $\left(\hat{a}_{1}, \hat{a}_{2}\right)$, having $\left(1, \sigma_{\hat{a}(2,1)} \sigma_{\hat{a}(1,1)}^{-1}\right)$ as direction vector. This line intersects the ellipse at the two points where the normal of the ellipse is directed along the $a_{1}$-axis. Note that the point $\left(a_{1}, \hat{a}_{2 \mid 1}\right)$ moves along this line when $a_{1}$ is varied. With (17) we are now in the position to construct the following two bounds for the two ambiguities $a_{1}$ and $a_{2}$,

$$
\left\{\begin{array}{l}
\left(\hat{a}_{1}-a_{1}\right)^{2} \leq \sigma_{\hat{a}(1,1)} \chi^{2} \\
\left(\hat{a}_{2 \mid 1}-a_{2}\right)^{2} \leq \sigma_{\hat{a}(2|1,2| 1)} \lambda\left(a_{1}\right) \chi^{2},
\end{array}\right.
$$

with $\lambda\left(a_{1}\right)=1-\left(\hat{a}_{1}-a_{1}\right)^{2} / \chi^{2} \sigma_{\hat{a}(1,1)}$. The corresponding intervals and their lengths are also shown in figure 1.

Our search for the integer least-squares ambiguities now proceeds as follows. First one selects an integer ambiguity

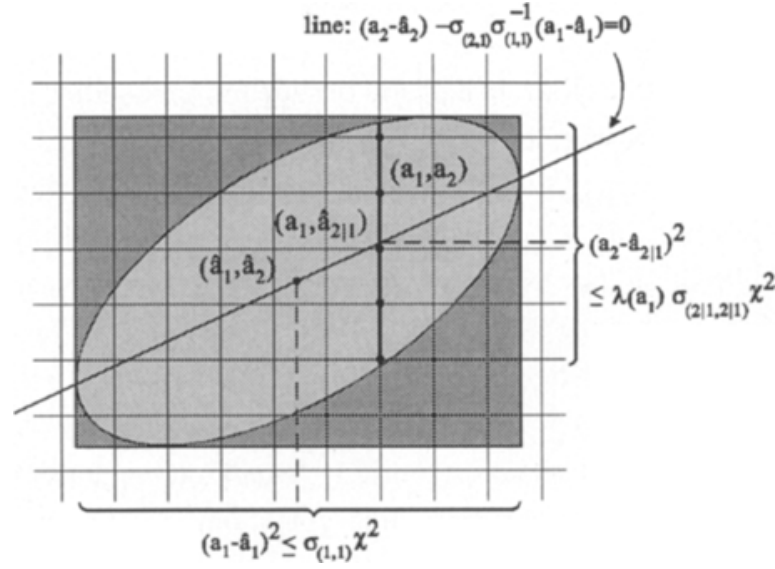

Figure 1: Ambiguity search space and bounds.

$a_{1}$ that satisfies the first bound of (18). It will be assumed that the two ambiguities are ordered such that one starts with the most precise least-squares ambiguity, i.e. that $\sigma_{\hat{a}(1,1)} \leq \sigma_{\hat{a}(2,2)}$. Then, based on this chosen integer ambiguity value $a_{1}$, the conditional least-squares estimate $\hat{a}_{2 \mid 1}$ and scalar $\lambda\left(a_{1}\right)$ are computed. These values are then used to select an integer ambiguity $a_{2}$ that satisfies the second bound of (18). Since we aim at finding the integer minimizer, it is natural to choose the integer candidates in such a way that the individual squares in the sum-of-squares (17) are made as small as possible. This implies that $a_{2}$ should always be chosen as the integer nearest to $\hat{a}_{2 \mid 1}$. But remember that $\hat{a}_{2 \mid 1}$ depends on $a_{1}$. For $a_{1}$ one first chooses the integer nearest to $\hat{a}_{1}$. If one then fails to find an integer $a_{2}$ that satisfies the second bound, one restarts and chooses for $a_{1}$ the second nearest integer to $\hat{a}_{1}$, and so on. Note that in this way, one is roughly following the direction of the line $\left(a_{1}, \hat{a}_{2 \mid 1}\right)$, working with $a_{1}$ along the $a_{1}$-axis from the inside of the ellipse, in an alternating fashion, towards the bounds of the ellipse. And this is continued until an admissible integer-pair $\left(a_{1}, a_{2}\right)$ is found, i.e. until a gridpoint is found that lies inside the ellipse. Then a shrinking of the ellipse is applied, by applying an appropriate downscaling of $\chi^{2}$, after which one continues with the next and following nearest integers to $\hat{a}_{1}$. This process is continued until one fails to find an admissible integer for $a_{1}$. The last found integer-pair is then the sought for integer least-squares solution.

Generalizing the above to the $n$-dimensional case, results in the following bounds for the individual ambiguities,

$$
\left(\hat{a}_{i \mid I}-a_{i}\right)^{2} \leq \sigma_{\hat{a}(i|l, i| \mid)} \lambda\left(a_{I}\right) \chi^{2}, i=1, \ldots, n,
$$

with $\lambda\left(a_{f}\right)=\left(1-\sum_{j=1}^{i-1}\left(\hat{a}_{j \mid J}-a_{j}\right)^{2} / \chi^{2} \sigma_{\hat{a}(j|, J j|, \mid}\right)$. These bounds are now used, quite analogous to the two dimensional case, for the search of the integer least-squares ambiguities. Note that 
the bounds of (19) are sharper than those that would result

when using the ellipsoidal planes of support for bounding the individual ambiguities. In that case, one would have: $\left(\hat{a}_{i}-a_{i}\right)^{2} \leq \sigma_{\hat{a}(i, i)} \chi^{2}$ for $i=1, \ldots, n$. And clearly, since $0 \leq \lambda\left(a_{i}\right) \leq 1$ and since a conditional variance is always smaller or at least as small as its unconditional counterpart, it follows that the bounds of (19) are always sharper or at least as sharp as $\sigma_{\hat{a}(i, i)} \chi^{2}$.

To conclude this section, we briefly remark on the practical implementation of the sequential conditional least-squares estimation. In the previous section, see (14), the correspondence was shown between the $L D L^{*}$-decomposition of $Q_{\grave{a}}$ and the sequential conditional least-squares estimation. It will be clear that the $L D L^{*}$-decomposition is easily constructed from the triangular Cholesky-decomposition. Many geodetic least-squares adjustment software-packages make use of the Cholesky-decomposition, but it is usually the Cholesky-decomposition of the normal matrix, $Q_{\hat{a}}^{-1}$, that is standard available and not the Cholesky-decomposition of $Q_{\hat{a}}$. This however, does not pose any difficulty. From the Cholesky-decomposition of the normal matrix, the $L D L^{*}$ decomposition of $Q_{a}^{-1}$ is easily constructed. Inversion gives then $Q_{\hat{a}}=L^{-*} D^{-1} L^{-1}$. This decomposition of $Q_{\hat{a}}$ is again unique. The difference with the decomposition of $Q_{\hat{a}}$ in (14) is however, that matrix $L^{-*}$ in $Q_{\hat{a}}=L^{-*} D^{-1} L^{-1}$ is uppertriangular and not lower-triangular. Hence, this decomposition would follow if one would apply a "backward" triangular decomposition to $Q_{\hat{a}}$. That is, instead of starting with $a_{1}$, one then starts with $a_{n}$. The conclusion reads therefore, that when one starts from a triangular decomposition of the normal matrix $Q_{\hat{a}}^{-1}$, bounds for the ambiguities can be constructed as easily as those of (19). The only difference would then be, that these bounds would correspond to a sequential conditional least-squares estimation that commences with a conditioning on the last ambiguity and stops with a conditioning on the first ambiguity.

\subsection{The GPS Spectrum of Ambiguity Conditional Variances}

In the previous section it was shown how we perform the search for the integer least-squares ambiguities. But as it was pointed out, it may happen that the search halts before a complete integer ambiguity candidate vector has been found. This occurs when the size of the bound is such that no candidate integer lies within the interval. The problem that the search for the integer least-squares ambiguities halts is a very serious one in case of GPS carrier-phase processing, in particular when short observational timespans are used. As it will be explained, this problem is intrinsically related to the structure of the GPS carrier-phase model of observation equations and the chosen parametrization.

For a single baseline model, it can be shown that the spectrum of ambiguity conditional variances, $\sigma_{\hat{a}(i|I, i| I)}$ for $i=1, \ldots, n$, has a large discontinuity when passing from the third conditional variance $\sigma_{\hat{a}(3|2,1 ; 3| 2,1)}$ to the fourth conditional variance $\sigma_{\hat{a}(4|3,2,1 ; 4| 3,2,1)}$. In order to explain this behaviour of the spectrum of conditional variances, the following example considers a synthetic 2-by-2 ambiguity variance-covariance matrix. The structure of this matrix is chosen such, that it resembles the structure of the actual $n$ by- $n$ ambiguity variance-covariance matrices.

\section{Example 1}

Let the variance-covariance matrix of the two least-squares ambiguities $\hat{a}_{1}$ and $\hat{a}_{2}$ be given as

$$
\left(\begin{array}{ll}
\sigma_{\hat{a}(1,1)} & \sigma_{\hat{a}(1,2)} \\
\sigma_{\hat{a}(2,1)} & \sigma_{\hat{a}(2,2)}
\end{array}\right)=\sigma^{2}\left(\begin{array}{ll}
1 & 0 \\
0 & 1
\end{array}\right)+\left(\begin{array}{l}
\beta_{1} \\
\beta_{2}
\end{array}\right)\left(\begin{array}{l}
\beta_{1} \\
\beta_{2}
\end{array}\right)^{*} .
$$

It will be assumed that

$$
\sigma^{2}<<\beta_{1}^{2}, \beta_{2}^{2} ; \beta_{1}^{2} \cong \beta_{2}^{2} .
$$

Note that the above 2-by-2 matrix is given as the sum of a scaled rank- 2 matrix and a rank-1 matrix. And because of (21), the entries of the rank- 2 matrix are very much smaller than the entries of the rank-1 matrix.

First we consider the correlation between the two ambiguities $\hat{a}_{1}$ and $\hat{a}_{2}$. It follows from (20) that the square of the correlation coefficient is given as

$$
\rho^{2}=\left(\left(1+\sigma^{2} / \beta_{1}^{2}\right)\left(1+\sigma^{2} / \beta_{2}^{2}\right)\right)^{-1} .
$$

Together with (21) this shows that $p^{2} \cong 1$. Hence, the two ambiguities are very heavily correlated. As a consequence of this extreme correlation, one will observe a large discontinuity in the conditional variances. To show this, consider the variance $\sigma_{\hat{a}(1,1)}$ and the conditional variance $\sigma_{\hat{a}(2|1,2| 1)}$. It follows from (20) that

$$
\sigma_{\hat{a}(1,1)}=\sigma^{2}+\beta_{1}^{2} ; \sigma_{\hat{a}(\{|1,2| 1)}=\sigma^{2}+\beta_{2}^{2} \frac{\sigma^{2} / \beta_{1}^{2}}{1+\sigma^{2} / \beta_{1}^{2}} .
$$

Together with (21) this shows that $\sigma_{\hat{a}(2|1.2| 1)}<<\sigma_{\ddot{a}(1,1)}$. 
Hence, there is a tremendous drop in value when one goes from the variance of the first ambiguity to the conditional variance of the second ambiguity. With $\beta_{1}^{2}$ sufficiently large, we approximately have $\sigma_{\hat{a}(1,1)} \cong \beta_{1}^{2}$ and $\sigma_{\hat{a}(2|1,2| 1)} \cong 2 \sigma^{2}$. The important implication of this result for the search of the integer least-squares ambiguities, is the following. When $\sigma_{\hat{a}(1,1)}$ is large and $\sigma_{\hat{a}(2|1,2| 1)}$ is extremely small, the problem of search-halting will be significant. A large $\sigma_{\hat{a}(1,1)}$ implies namely, that the first bound of (18) will be rather loose. Quite a number of integers will therefore satisfy this first bound. This on its turn implies, when we go to the second bound, which is very tight due to $\sigma_{\hat{a}(2|1,2| 1)}<<\sigma_{\hat{a}(1,1)}$, that we have a high likelihood of not being able to find an integer that satisfies this second bound. The potential of halting is therefore very significant when one goes from the first to the second bound. As a consequence a large number of trials are required, before one is able to find a candidate integerpair.

The above two-dimensional example has shown, how the search is affected when the conditional variances show a large discontinuity. This effect is also typically experienced in the actual search for the integer least-squares ambiguities. The structure of (20) resembles namely the structure of the actual ambiguity variance-covariance matrices. In case of the single baseline model, the actual $n$-by- $n$ ambiguity variancecovariance matrices can also be written as the sum of two matrices. The first matrix in this sum is then of rank $n$, but contains very small entries because of the high precision with which the carrier-phases can be observed. The second matrix in the sum will be of rank 3 , and contains entries which are relatively large due to the poor precision with which the baseline can be estimated when the ambiguities are still estimated as reals. As a result of this structure, the actual spectrum of ambiguity conditional variances will generally show a large discontinuity after the third variance:

$$
\sigma_{\hat{a}(j|J, j| \jmath)}<<\sigma_{\hat{a}(i|I, i| I)} i=1,2,3, j=4, \ldots, n .
$$

This implies, since the first three bounds of (19) will be rather loose and the remaining bounds will be very tight due to the discontinuity, that the potential of halting will be significant when one passes from the third bound to the fourth bound. The following example gives a representative illustration of the characteristics of the spectrum of ambiguity conditional variances.

\section{Example 2}

This example is based on a 7 satellite configuration using dual frequency carrier-phase data for an observational timespan of two seconds. Figure 2 shows the spectrum of conditional standard deviations expressed in cycles. Note the logarithmic scale along the vertical axis.

Since 7 satellites were observed on both frequencies, we have twelve double-difference ambiguities and therefore also

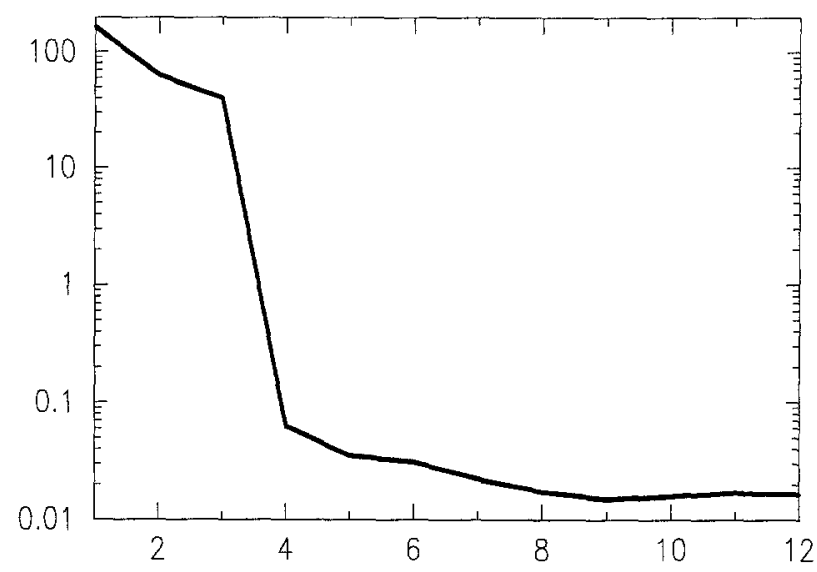

Figure 2: A GPS spectrum of conditional standard deviations.

twelve conditional standard deviations. The figure clearly shows the tremendous drop in value when passing from the third to the fourth standard deviation. There are three large conditional standard deviations and nine extremely small ones. The nine small conditional standard deviations are due to the presence of satellite redundancy and the fact that dual frequency carrier-phase data is observed. And it is because of the large values of the first three conditional standard deviations and the extreme small values for the remaining conditional standard deviations, that the search for the integer least-squares ambiguities will be rather timeconsuming.

It will be clear that the efficiency of the search for the integer least-squares ambiguities could be improved considerably, if we would be able to eliminate the discontinuity in the spectrum and lower the values of the large conditional standard deviations. In the next chapter it will be shown how this can be achieved through a reparametrization of the ambiguities.

\section{The Reparametrized Ambiguity Estimation Problem}

This chapter is devoted to the elimination of the potential problem of halting. The original integer least-squares problem is reparametrized such that an equivalent formulation is obtained, but one that is easier to solve. As our search procedure of the previous chapter, also the reparametrizing ambiguity transformation makes use of the 
sequential conditional least-squares estimation of the ambiguities. Although the aim is to fully decorrelate the ambiguities, true diagonality of the variance-covariance matrix will be difficult to reach. In section 3.1 it is shown that this is due to the fact that only a particular class of ambiguity transformations is admissible. Being restricted to this class, section 3.2 shows to what extent the ambiguities can be decorrelated for the two-dimensional case. Based on an integer approximation of the conditional least-squares transformation, a decorrelating two-dimensional ambiguity transformation is introduced. With this transformation it is guaranteed that the square of the correlation coefficient of the transformed ambiguities is less than or equal to $1 / 4$. In section 3.3 the $n$-dimensional problem is discussed. The discontinuity in the GPS-spectrum of conditional variances is flattened through a repeated use of the two-dimensional ambiguity transformation. By removing the discontinuity with the ambiguity transformation, transformed ambiguities are obtained that show a dramatic improvement in both precision and correlation. As a result the search for the transformed ambiguities can be performed in a highly efficient manner. In section 3.4 a measure for the gain in baseline precision due to ambiguity fixing is introduced. It is shown how this gain can be computed directly from the spectrum of conditional variances.

\subsection{The Class of Admissible Ambiguity Transformations}

Certain linear combinations of the GPS-observables play a prominent role in the problem of ambiguity fixing. For the purpose of ambiguity fixing one usually aims at those integer linear combinations that produce a phase observable which has a relatively long wavelength, a relatively low noise behaviour and a reasonable small ionospheric delay [20]. And these properties are indeed very beneficial to the ambiguity fixing process. At present however, all integer linear combinations that are considered, are restricted to the single-channel dual-frequency case. But this implies, that the relative receiver-satellite geometry, which is so emphatically present in the ambiguity variance-covariance matrix, is not taken into account in the current linear combinations. This observation suggests therefore, that it is of interest to consider multi-channel integer linear combinations, instead of only the current single-channel integer linear combinations.

In [19] the admissible class of multi-channel integer linear combinations of the ambiguities was identified. It was shown that a full-rank $n$-by- $n$ transformation $Z^{*}$ of the original ambiguity vector $a$ to a new ambiguity vector $z$, giving $z=$
$Z^{*} a$, is admissible if and only if $Z^{*}$ is volume preserving and has entries which all are integer. Typical examples of transformations that fall in this class are the identity matrix and the permutation matrices. But also all ambiguity transformations that change the choice of reference satellite in the double-difference ambiguities, are admissible. And on the single-channel level, the same holds for the transformation from the $L_{1}$ and $L_{2}$ ambiguity to the $L_{1}$ ambiguity and the wide-lane ambiguity. Note however, that the one-to-one single-channel transformation from the $L_{1}$ and $L_{2}$ ambiguity to the narrow-lane and wide-lane ambiguity is not admissible.

With the class of admissible ambiguity transformations identified, we can now try to use members from this class to aid the ambiguity fixing process. Let $Z^{*}$ be an ambiguity transformation, which is used to transform the ambiguities as

$$
z=Z^{*} a, \hat{z}=Z^{*} \hat{a}, Q_{\hat{z}}=Z^{*} Q_{\hat{a}} Z
$$

The ambiguity integer least-squares problem (10) would then transform accordingly into the equivalent minimization problem

$$
\min _{z}(\hat{z}-z)^{*} Q_{\hat{z}}^{-1}(\hat{z}-z) \text { with } z \in Z^{n}
$$

Similarly, the original ambiguity searchspace (16) would transform into the new ambiguity searchspace

$$
\sum_{i=1}^{n}\left(\hat{z}_{i \mid I}-z_{i}\right)^{2} / \sigma_{\hat{z}(i|I, i| l)} \leq \chi^{2}
$$

Note that this transformed searchspace not only has a volume which is identical to the volume of the original searchspace, but it also has, as it should be, the same number of candidate gridpoints. Based on the transformed searchspace (27), the search for the integer minimizer $\breve{z}$ of (26), can now be performed in exactly the same way as it has been described in section 2.4 for the original searchspace. And once the integer least-squares ambiguity $\check{z}$ has been found, the integer minimizer $\breve{a}$ of (10) can be recovered from invoking $\breve{a}=Z^{-*} \breve{z}$. The final baseline solution $\check{b}$ follows then from (11). Alternatively, one could also use

$$
\check{b}=\hat{b}-Q_{\hat{b} \hat{z}} Q_{\hat{z}}^{-1}(\hat{z}-\check{z})
$$

to obtain the final baseline solution.

In order to have any use for the ambiguity transformation $Z^{*}$, we should aim at finding a transformation that makes the transformed integer least-squares problem (26) easier to solve than the original problem (10). Clearly, the ideal situation would be, to have a transformation $Z^{*}$ that allows for a full 
decorrelation of the ambiguities. In that case, $Q_{\hat{z}}$ is diagonal and (26) can simply be solved by rounding the entries of $\hat{z}$ to their nearest integer. Unfortunately however, the restrictions on $Z$ $Z$ do generally not allow for a complete diagonalization of the variance-covariance matrix. For instance, the choice where $Z$ contains the (normalized) eigenvalues of $Q_{\hat{a}}$ is not allowed. Although this transformation preserves volume, it generally does not have entries which all are integer. Also a diagonalization based on $Z^{*}=L^{-1}$, with $L$ being the triangular factor of $Q_{\hat{a}}$, is not admissible. Again, although $L$ is volume-preserving, its nonzero off-diagonal entries will generally not be integer. This shows that in terms of diagonality, one will have to be content with a somewhat less perfect result. Nevertheless a decrease in correlation, although not complete, will already be very helpful, since it would close the existing gap between $\sigma_{\hat{a}(1,1)}$ and $\sigma_{\hat{a}(2|1,2| 1)}$ of example 1 . In the next section it will be shown to what extent the ambiguities can be decorrelated in the two-dimensional case.

\subsection{A 2D-Decorrelating Ambiguity Transformation}

In order to answer the question as to how to construct the ambiguity transformation $Z^{*}$, we first consider the problem in two dimensions. Let the ambiguities and their variancecovariance matrix be given as

$$
\hat{a}=\left(\begin{array}{l}
\hat{a}_{1} \\
\hat{a}_{2}
\end{array}\right) \text { and } Q_{\hat{a}}=\left(\begin{array}{ll}
\sigma_{\hat{a}(1,1)} & \sigma_{\hat{a}(1,2)} \\
\sigma_{\hat{a}(2,1)} & \sigma_{\hat{a}(2,2)}
\end{array}\right) \text {. }
$$

From section 2.3 we already know, that the conditional leastsquares based transformation returns least-squares ambiguities that are fully decorrelated. When (13) is written in vector-matrix form, we obtain for the two-dimensional case, the transformation

$$
\left(\begin{array}{l}
\hat{a}_{1} \\
\hat{a}_{2 \mid 1}
\end{array}\right)=\left(\begin{array}{cc}
1 & 0 \\
-\sigma_{\hat{a}(2,1)} \sigma_{\hat{a}(1,1)}^{-1} & 1
\end{array}\right)\left(\begin{array}{l}
\hat{a}_{1} \\
\hat{a}_{2}
\end{array}\right) .
$$

Since we are studying the effect of transformations on $Q_{\tilde{a}}$, we have for reasons of convenience skipped the elements $a_{1}$ and $a_{2}$ in the above transformation. Note, that this transformation not only decorrelates, but, in line with the correspondence between linear least-squares estimation and best linear unbiased estimation, also returns $\hat{a}_{2 \mid 1}$ as the element which has the best precision of all linear unbiased functions of $\hat{a}_{1}$ and $\hat{a}_{2}$. Also note, that the above transformation is volume-preserving. Hence, the only condition that prohibits the above transformation from becoming an admissible ambiguity transformation is, that not all entries of the transformation are integer. But, this shortcoming is easily repaired. We simply approximate the above transformation by replacing $\sigma_{\hat{a}(2,1)} \sigma_{\hat{a}(1,1)}^{-1}$ by $\left[\sigma_{\hat{a}(2,1)} \sigma_{\hat{a}(1,1)}^{-1}\right]$, where [.] stands for "rounding to the nearest integer". This gives

$$
\left(\begin{array}{l}
\hat{a}_{1} \\
\hat{a}_{2^{\prime}}
\end{array}\right)=\left(\begin{array}{cc}
1 & 0 \\
-\left[\sigma_{\hat{a}(2,1)} \sigma_{\hat{a}(1,1)}^{-1}\right] & 1
\end{array}\right)\left(\begin{array}{l}
\hat{a}_{1} \\
\hat{a}_{2}
\end{array}\right) .
$$

The volume-preserving property is retained by this integer approximation. But the full decorrelation property is of course not retained. What remains to be verified therefore, is whether the above transformation still allows us to reduce the correlation between the ambiguities. In order to verify this, we first assume, as was done in our search algorithm of section 2.4, that the two ambiguities are ordered such that $\sigma_{\hat{a}(1,1)} \leq \sigma_{\hat{a}(2,2)}$. This implies that $\left|\sigma_{\hat{a}(2,1)} \sigma_{\hat{a}(1,1)}^{-1}\right| \geq\left|\sigma_{\hat{a}(1,2)} \sigma_{\hat{a}(2,2)}^{-1}\right|$. We also assume that $\sigma_{\hat{a}(2,1)} \sigma_{\hat{a}(1,1)}^{-1} \notin(-1 / 2,+1 / 2]$. Because, otherwise we would have $\left[\sigma_{\hat{a}(2,1)} \sigma_{\hat{a}(1,1)}^{-1}\right]=0$, which would mean that the ambiguity transformation (31) reduces to the trivial identity transformation. Also an interchange of the two ambiguities would then not help, because if $\left[\sigma_{\hat{a}(2,1)} \sigma_{\hat{a}(1,1)}^{-1}\right]=0$, then also $\left[\sigma_{\hat{a}(1,2)} \sigma_{\hat{a}(2,2)}^{-1}\right]=0$, since $\left|\sigma_{\hat{a}(2,1)} \sigma_{\hat{a}(1,1)}^{-1}\right| \geq\left|\sigma_{\hat{a}(1,2)} \sigma_{\hat{\alpha}(2,2)}^{-1}\right|$.

It follows from the volume-preserving property of the ambiguity transformation that the determinant of the variance-covariance matrix of the original ambiguities $\hat{a}_{1}$ and $\hat{a}_{2}$ is identical to the determinant of the variance-covariance matrix of the transformed ambiguities $\hat{a}_{1}$ and $\hat{a}_{2^{\prime}}$. We therefore have the following equality of determinants

$$
\sigma_{\hat{a}(1,1)} \sigma_{\hat{a}(2,2)}\left(1-\rho^{2}\right)=\sigma_{\hat{a}(1.1)} \sigma_{\hat{a}\left(2^{\prime}, 2\right)}\left(1-\rho^{2}\right) .
$$

From this equality we conclude that the correlation decreases whenever the variance of the transformed ambiguity $\hat{a}_{2^{\prime}}$ is smaller than the variance of the original ambiguity $\hat{a}_{2}$. Hence, by decreasing the variance we automatically reach a decrease in correlation, $\rho^{2}<\rho^{2}$, and vice versa. Note, that this coupling between variance and correlation is a direct consequence of the volume-preserving property of the ambiguity transformation. We already know that the conditional least-squares estimate $\hat{a}_{2 \mid 1}$ has the smallest variance attainable. What remains to be shown is therefore whether its integer approximation $\hat{a}_{2^{\prime}}$ also has a variance that is smaller than the variance of $\hat{a}_{2}$. In order to show this, we express $\left(\hat{a}_{1}, \hat{a}_{2^{\prime}}\right)$ in terms of $\left(\hat{a}_{1}, \hat{a}_{2 \mid 1}\right)$. With (31) and the inverse of (30), this gives

$$
\left(\begin{array}{l}
\hat{a}_{1} \\
\hat{a}_{2^{\prime}}
\end{array}\right)=\left(\begin{array}{ll}
1 & 0 \\
\varepsilon & 1
\end{array}\right)\left(\begin{array}{l}
\hat{a}_{1} \\
\hat{a}_{2 \mid 1}
\end{array}\right) \text {, with }|\varepsilon| \leq 1 / 2 .
$$


Application of the error propagation law gives then

$$
\left\{\begin{array}{l}
\sigma_{\hat{a}(1,2)}=\varepsilon \sigma_{\hat{a}(1,1)} \\
\sigma_{\hat{a}\left(2^{\prime}, 2^{\prime}\right)}=\varepsilon^{2} \sigma_{\hat{a}(1,1)}+\left(1-\rho^{2}\right) \sigma_{\hat{a}(2,2)} .
\end{array}\right.
$$

For the second equation we may also write $\sigma_{\hat{a}(2,2)}=\sigma_{\hat{a}(2,2)}-\left(\sigma_{\hat{a}(2,1)}^{2} \sigma_{\hat{a}(1,1)}^{-2}-\varepsilon^{2}\right) \sigma_{\hat{a}(1,1)}$. This shows, since $\sigma_{\hat{a}(2,1)}^{2} \sigma_{\hat{a}(1,1)}^{-2} \geq 1 / 4$ and $\varepsilon^{2} \leq 1 / 4$ that $\sigma_{\hat{a}\left(2^{\prime}, 2\right)} \leq \sigma_{\hat{a}(2,2)}$, with the equality only when $\sigma_{\hat{a}(2,1)} \sigma_{\tilde{a}(1,1)}^{-1}=-1 / 2$. The conclusion reads therefore, that the ambiguity transformation (31) indeed allows us to decrease the correlation between the two ambiguities.

So far, we have only dealt with one of the two ambiguities, namely $a_{2}$. But now that $\hat{a}_{2}$ has been transformed into $\hat{a}_{2}$, we could think of interchanging the role of the two ambiguities and proceed with transforming the first ambiguity $\hat{a}_{1}$ into $\hat{a}_{1^{\prime}}$. The corresponding ambiguity transformation reads then

$$
\left(\begin{array}{l}
\hat{a}_{1^{\prime}} \\
\hat{a}_{2^{\prime}}
\end{array}\right)=\left(\begin{array}{cc}
1 & -\left[\sigma_{\hat{a}(1,2)} \sigma_{\hat{a}\left(2^{\prime}, 2\right)}^{-1}\right] \\
0 & 1
\end{array}\right)\left(\begin{array}{l}
\hat{a}_{1} \\
\hat{a}_{2^{\prime}}
\end{array}\right) .
$$

This transformation makes only sense however, when

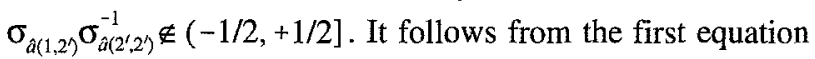
of (34) that $\sigma_{\hat{a}(1,2)} \sigma_{\hat{a}\left(2^{\prime}, 2^{\prime}\right)}^{-1}=\varepsilon \sigma_{\hat{a}(1,1)} \sigma_{\hat{a}\left(2^{\prime}, 2^{\prime}\right)}^{-1}$. We therefore have $\sigma_{\hat{a}(1,2)} \sigma_{\hat{a}\left(2^{\prime}, 2\right)}^{-1} \in(-1 / 2,+1 / 2]$ and thus $\left[\sigma_{\hat{a}(1,2)} \sigma_{\tilde{a}_{(2,2}, 2}^{-1}\right]=0$ whenever $\sigma_{\tilde{a}\left(2^{\prime}, 2\right\}} \geq \sigma_{\hat{a}(1,1)}$. This shows that it only makes sense to continue when $\sigma_{a\left(2^{\prime}, 2\right)}<\sigma_{\hat{a}(1,1)}$. And when one continues, one again will be able to obtain a further decrease in the correlation between the two ambiguities. In fact, one can proceed in this way and construct a concatenated form of ambiguity transformations $Z^{*}$, in which each individual transformation contributes to a lessening of the correlation. The last ambiguity transformation in this string of transformations will then reduce to the trivial identity transformation. And when this happens, we would have for the transformed ambiguities, denoted as $\hat{z}_{1}$ and $\hat{z}_{2}$, that $\left[\sigma_{\tilde{z}(2,1)} \sigma_{\tilde{z}(1,1)}^{-1}\right]=0$ and $\left[\sigma_{\tilde{z}(1,2)} \sigma_{\tilde{z}(2,2)}^{-1}\right]=0$, or that $\left|\sigma_{\tilde{z}(2,1)} \sigma_{\tilde{z}(1,1)}^{-1}\right|$ $\leq 1 / 2$ and $\left|\sigma_{\tilde{z}(1,2)} \sigma_{\tilde{z}(2,2)}^{-1}\right| \leq 1 / 2$, from which it follows that

$$
\rho_{\hat{z}}^{2}=\frac{\sigma_{\hat{z}(1,2)}^{2}}{\sigma_{\hat{z}(1,1)} \sigma_{\hat{z}(2,2)}} \leq 1 / 4 .
$$

This is an important result, because it shows that we can transform the original ambiguities, which are usually highly correlated, into new ambiguities which, are much less correlated and which also, because of the volume-preserving property, see (32), are more precise. From the above bound it follows, together with $\sigma_{z(2|1,2| 1)}=\left(1-\rho_{\tilde{z}}^{2}\right) \sigma_{z(2,2)}$ and $\sigma_{\hat{z}(1,1)}$ $\leq \sigma_{\tilde{z}(2,2)}$ that

$$
\sigma_{\tilde{z}(2|1,2| 1)} \geq \frac{3}{4} \sigma_{\tilde{z}(1,1)} .
$$

Hence, the ambiguity transformation $Z^{*}$ guarantees that the transformed conditional variance $\sigma_{\hat{z}(2|1,2| 1)}$ will never be much smaller than the variance $\sigma_{\tilde{z}(1,1)}$. But this implies, that $Z^{*}$ indeed removes to a large extent any discontinuity present in the original conditional variances, $\sigma_{\hat{a}(2|1,2| 1)}<<\sigma_{\hat{a}(1,1)}$.

Geometrically, the above constructed sequence of ambiguity transformations can be given the following useful interpretation (see figure 3). Imagine the original two-
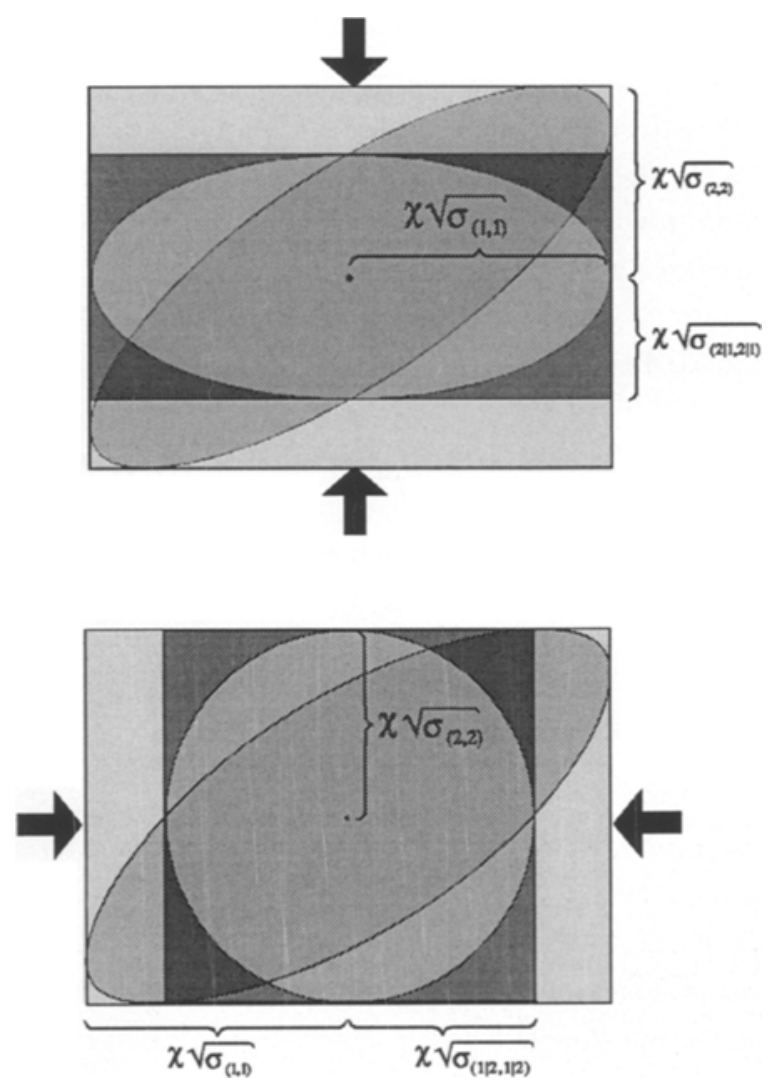

Figure 3: Decorrelating ambiguities by pushing tangents.

dimensional ambiguity searchspace centred at $\hat{a}$. A full decorrelation between the two ambiguities can be realized, if we push the two horizontal tangents of the ellipse from the $\pm\left(\sigma_{\hat{a}(2,2)} \chi^{2}\right)^{1 / 2}$ level towards the $\pm\left(\sigma_{\hat{a}(2|1,2| 1)} \chi^{2}\right)^{1 / 2}$ level, while at the same time keeping fixed the volume (area) of the ellipse and the location of the two vertical tangents. Alternatively, one can also achieve a full decorrelation, if instead of the two horizontal tangents, the two vertical tangents are pushed from the $\pm\left(\sigma_{\hat{a}(1,1)} \chi^{2}\right)^{1 / 2}$ level towards the $\pm\left(\sigma_{\hat{a}(1|2,1| 2)} \chi^{2}\right)^{1 / 2}$ level. Unfortunately however, both these transformations are not admissible, since it is not guaranteed that they result in integer ambiguities. Therefore, instead of using these two 
transformations, we make use of their integer approximations. The first transformation (31) then pushes the two horizontal tangents of the ellipse from the $\pm\left(\sigma_{\hat{a}(2,2)} \chi^{2}\right)^{1 / 2}$ level towards the $\pm\left(\sigma_{\hat{a}\left(2^{\prime}, 2\right.} \chi^{2}\right)^{1 / 2}$ level, while at the same time keeping fixed the volume of the ellipse and the location of the two vertical tangents of the ellipse. The second transformation (35) then pushes the two vertical tangents from the $\pm\left(\sigma_{\hat{\alpha}(1,1)} \chi^{2}\right)^{1 / 2}$ level towards the $\pm\left(\sigma_{\hat{a}\left(1^{\prime}, 1\right)} \chi^{2}\right)^{1 / 2}$ level, while at the same time keeping fixed the volume of the ellipse and the location of the two horizontal tangents. And this process is continued until the next transformation reduces to the trivial identity transformation. Since the volume of the ellipse is kept constant at all times, whereas the volume of the enclosing box is reduced in each step, it also follows that the ellipse is forced to become more sphere-like.

Based on the variance-covariance matrix of example 1, the following example illustrates how the ambiguity transformation $Z^{*}$ succeeds in decorrelating the two ambiguities.

\section{Example 3}

When the values

$$
\sigma=0.2, \beta_{1}=5.0 \text { and } \beta_{2}=6.0
$$

are substituted into (20), we obtain

$$
Q_{\hat{a}}=\left(\begin{array}{cc}
25.04 & 30 \\
30 & 36.04
\end{array}\right) \text {. }
$$

From this it follows that the two ambiguities are highly correlated and that the with (39) corresponding ambiguity search space is very elongated. The correlation coefficient $\rho_{\hat{a}}$ and elongation $e_{\hat{a}}$ read

$$
\rho_{\hat{a}}=0.999 \text { and } e_{\hat{a}}=39.064 \text {. }
$$

Elongation is measured as the ratio of the lengths of the major and minor semi-axes of the ambiguity search ellipse. If we now apply our method of ambiguity decorrelation, the corresponding ambiguity transformation becomes

$$
Z^{*}=\left(\begin{array}{rr}
6 & -5 \\
-1 & 1
\end{array}\right)
$$

The variance-covariance matrix of the transformed ambiguities reads therefore

$$
Q_{\hat{\imath}}=\left(\begin{array}{rr}
2.44 & -0.44 \\
-0.44 & 1.08
\end{array}\right) \text {. }
$$

This result shows, that the transformed ambiguities are indeed less correlated, more precise, and also have an ambiguity search space that is less elongated:

$$
\rho_{\hat{z}}=-0.271 \text { and } e_{\bar{z}}=1.645 \text {. }
$$

And because of this result, the gap in the conditional variances has been largely removed: $\sigma_{\hat{a}(1,1)}=25.04, \sigma_{\hat{a}(2|1,2| 1)}=$ 0.098 versus $\sigma_{\hat{\imath}(1,1)}=2.44, \sigma_{\hat{\imath}(2|1,2| 1)}=1.00$. Also note that the determinant of the variance-covariance matrix remained invariant under the ambiguity transformation. Both $Q_{\hat{a}}$ and $Q_{\hat{z}}$ have a determinant equal to 2.442 . This invariance is due to the volume-preserving property of (41).

\subsection{Flattening the Spectrum of Ambiguity Conditional Variances}

As it was shown in section 2.5 , it is the large discontinuity in the spectrum of ambiguity conditional variances, that forms a hindrance for the efficient search of the integer leastsquares ambiguities. A flattened spectrum will therefore be very beneficial indeed for our search. Having a flattened spectrum automatically implies, when $n>3$, that the three large variances in the spectrum get significantly smaller. This can be seen as follows. Since the ambiguity transformation is volume-preserving, the determinant of the ambiguity variance-covariance matrix remains invariant under the transformation. Hence, since the determinant of the lower triangular matrix $L$ in (14) equals one, it follows that also the determinant of the diagonal matrix $D$ and therefore the product of all conditional variances remains invariant under the transformation. Compare with (32) of section 3.2. But this implies, that by flattening the spectrum, the very small conditional variances in the spectrum automatically allow us to significantly decrease the three large conditional variances. And as a result of this, our search for the integer leastsquares ambiguities would then already commence with a very tight bound, thus assuring with a very high likelihood that the first chosen integer candidate is indeed already the one sought.

The fact that the very small conditional variances in the spectrum allow us to significantly decrease the three large conditional variances, now also makes quite clear what role is played by satellite redundancy and dual frequency data. When both are absent, we have $n=3$. In that case, the 
absence of very small conditional variances prohibits us to a high degree from "pulling down" the large variances in the spectrum. In case of satellite redundancy and/or dual frequency data however, we have $n>3$. Now the presence of very small conditional variances does allow us to bring the large variances down to much smaller values. And the larger $n-3$ is, the more we are able to bring the flattened spectrum to a lower level.

In the previous section it was shown how to decorrelate the two ambiguities, thereby removing the gap between $\sigma_{\hat{a}(1,1)}$ and $\sigma_{\hat{a}(2 \mid 1,2 ! 1)}$. The two-dimensional ambiguity transformation was constructed from a sequence of transformations of the following two types:

$$
Z_{1}^{*}=\left(\begin{array}{cc}
1 & 0 \\
z_{21} & 1
\end{array}\right) \text { and } Z_{2}^{*}=\left(\begin{array}{cc}
1 & z_{12} \\
0 & 1
\end{array}\right),
$$

in which $z_{21}$ and $z_{12}$ are appropriately chosen integers. To generalize this to the $n$-dimensional case, we first need to generalize these type of transformations accordingly. Here however, we are faced with a difficulty, since one can think of different generalizations all of which reduce to one of the above when $n=2$. One rather straightforward generalization follows however, when one considers the bounding of the triangular factor that is achieved by $Z_{1}^{*}$ for $z_{21}=-\left[\sigma_{\hat{a}(2,1)} \sigma_{\hat{a}(1,1)}^{-1}\right]$. From an application of the error propagation law to (33) follows namely

$$
\left(\begin{array}{ll}
\sigma_{\hat{a}(1,1)} & \sigma_{\hat{a}(1,2)} \\
\sigma_{\hat{a}\left(2^{\prime}, 1\right)} & \left.\sigma_{\hat{a}\left(2^{\prime}, 2\right)}\right)
\end{array}\right)=\left(\begin{array}{ll}
1 & 0 \\
\varepsilon & 1
\end{array}\right)\left(\begin{array}{cc}
\sigma_{\hat{a}(1,1)} & 0 \\
0 & \sigma_{\hat{a}(2|1,2| 1)}
\end{array}\right)\left(\begin{array}{ll}
1 & 0 \\
\varepsilon & 1
\end{array}\right)^{*},
$$

with $|\varepsilon| \leq 1 / 2$. In order to generalize this result to dimensions higher than two, we start from $Q_{\hat{a}}=L D L^{*}$. Note that if the entries of $L$ would be integer, then so would the entries of its inverse $L^{-1}$ be. In fact, the inverse $L^{-1}$, being integer and volume-preserving, would then be the perfect candidate to diagonalize $Q_{\hat{a}}$. One would then be able to fully decorrelate all ambiguities in just one step. This observation suggests, even though the entries of $L$ will be non-integer in general, that we choose the $n$-by- $n$ matrix $Z_{1}^{*}$ as a lower triangular matrix, with integer entries and with one's on its diagonal. Matrix $Z_{1}^{*}$ can then be constructed from subtracting suitable integer multiples of the first $(i-1)$ rows of $L$ from row $i$ of $L$ for $i=n, \ldots, 2$. Using this matrix $Z_{1}^{*}$, one obtains the triangular decomposition $Z_{1}^{*} Q_{\hat{a}} Z_{1}=\left(Z_{1}^{*} L\right) D\left(Z_{1}^{*} L\right)^{*}$, in which, in analogy with (45), the absolute values of all non-diagonal entries of $Z_{1}^{*} L$ are guaranteed to be bounded by a half. This bounding of the triangular factor $Z_{1}^{*} L$ implies, when the non- diagonal entries of $L$ are larger than a half in absolute value, that the precision of all but the first transformed ambiguity will be improved. And this improvement will be more noteworthy, the more the non-diagonal entries of $L$ are decreased in size.

Although the above bounding of the triangular factor does allow for some decorrelation and some improvement of precision, one should note however, that all conditional variances remain invariant under the transformation $Z_{1}^{*}$. Hence, not only the variance $\sigma_{\hat{a}(1,1)}$ remains large, but the complete spectrum of ambiguity conditional variances, including its discontinuity, remains untouched. One should therefore not expect too much from the single transformation $Z_{1}^{*}$. This suggests, in analogy with the two-dimensional case, that one tries, in one way or another, to set up a sequence of $n$-by- $n$ transformations. But here, the problem of dimension takes its revenge in the sense that one can try to pursue many different alternatives. Fortunately, there is no need to follow this line of thought, since excellent results can already be obtained when we keep ourselves to the two-dimensional ambiguity transformation of the previous section and apply it to the $n$-dimensional case as well.

Transformations of the type (44) are known as Gausstransformations and they are considered to be the basic tools for zeroing entries in matrices [25]. In our case, due to the integer nature of $z_{12}$ and $z_{21}$, they will be used to decrease the conditional correlations instead of zeroing them, thereby trying to flatten the spectrum of ambiguity conditional variances. In order to see how we can construct the overall $n$-dimensional ambiguity transformation on the basis of the two-dimensional transformation of the previous section, consider the discontinuity in the spectrum. The discontinuity is located at the two neighbouring conditional variances $\sigma_{\hat{a}(i|, i,| l)}$ and $\sigma_{\hat{a}(i+1[I+1, i+1 \mid I+1)}$ for $i=3$. Hence, if we let $\hat{a}_{i \mid I}$ and $\hat{a}_{i+1 \mid i}$, for $i=3$, play the role of our two ambiguities $\hat{a}_{1}$ and $\hat{a}_{2}$ of the previous section, we should be able to remove this discontinuity from the spectrum by using the decorrelating two-dimensional ambiguity transformation of the previous section. Thus, instead of applying the twodimensional ambiguity transformation to the unconditional least-squares ambiguities, it is applied to the conditional least-squares ambiguities. This would then give, in analogy to (37),

$$
\sigma_{\hat{z}(i+1|I+1, i+1| I+1)} \geq \frac{3}{4} \sigma_{\hat{z}(i|l, i|)},
$$

for $i=3$. Note, that as a consequence of the fact that we are transforming conditional least-squares ambiguities, the other conditional variances remain unaffected by this 
transformation. Now, in order to construct and apply the two-dimensional transformation, we need the variancecovariance matrix of $\hat{a}_{i \mid l}$ and $\hat{a}_{i+1 \mid l}$. In addition, we also need to know how the transformation affects the variancecovariance matrix $Q_{\hat{a}}$. We will therefore again make use of the sequential conditional least-squares decomposition (14).

To consider what happens to (14) when the two-dimensional ambiguity transformation is applied to $\hat{a}_{i}$ and $\hat{a}_{i+1}$, we partition the lower triangular matrix $L$ and diagonal matrix $D$ of $Q_{\hat{a}}=L D L^{*}$ as

$$
L=\left(\begin{array}{lll}
L_{11} & & \\
L_{21} & L_{22} & \\
L_{31} & L_{32} & L_{33}
\end{array}\right) \text { and } D=\left(\begin{array}{lll}
D_{11} & & \\
& D_{22} & \\
& & D_{33}
\end{array}\right)
$$

in which $L_{11}$ is of order (i-1), $L_{22}$ is of order 2 and $L_{33}$ is of order $(n-i-1)$. The dimensions of the other submatrices in $L$ and $D$ are determined accordingly. Note that $\left(L_{21} D_{11} L_{21}{ }^{*}+\right.$ $\left.L_{22} D_{22} L_{22}{ }^{*}\right)$ is the variance-covariance matrix of the leastsquares ambiguities $\hat{a}_{i}$ and $\hat{a}_{i+1}$, whereas $L_{22} D_{22} L_{22}{ }^{*}$ is the variance-covariance matrix of the conditional least-squares ambiguities $\hat{a}_{i \mid l}$ and $\hat{a}_{i+1 \mid \Gamma}$. It is this last matrix that is now used for the construction of the two-dimensional ambiguity transformation. Hence, the conditional variance-covariance matrix $L_{22} D_{22} L_{22}{ }^{*}$ now plays the role of the variancecovariance matrix in (29). The two-dimensional ambiguity transformation will be denoted as $Z_{22}{ }^{*}$. With $Z_{22}{ }^{*}$ determined from $L_{22} D_{22} L_{22}{ }^{*}$, we can $Z_{22}{ }^{*}$-transform the unconditional least-squares ambiguities $\hat{a}_{i}$ and $\hat{a}_{i+1}$, while leaving the other least-squares ambiguities unchanged. As a result we obtain the transformed ambiguity vector $\hat{a}^{\prime}=\left(\hat{a}_{1}, . ., \hat{a}_{i-1}, \hat{z}_{i}, \hat{z}_{i+1}, \hat{a}_{i+2}\right.$, .., $\left.\hat{a}_{n}\right)^{*}$, with corresponding variance-covariance matrix $Q_{\hat{a}^{\prime}}$. This matrix will have a new triangular-decomposition $Q_{\hat{a}^{\prime}}=L^{\prime} D^{\prime} L^{\prime}$, with

$$
L^{\prime}=\left(\begin{array}{lll}
L_{11} & & \\
\overline{L_{21}} & \overline{L_{22}} & \\
L_{31} & \bar{L}_{32} & L_{33}
\end{array}\right) \text { and } D^{\prime}=\left(\begin{array}{lll}
D_{11} & & \\
& \bar{D}_{22} & \\
& & D_{33}
\end{array}\right)
$$

and where

$$
\left\{\begin{aligned}
\overline{L_{21}} & =Z_{22}^{*} L_{21} \\
\overline{L_{22}} \overline{D_{22}} \bar{L}_{22}^{*} & =Z_{22}^{*}\left(L_{22} D_{22} L_{22}^{*}\right) Z_{22} \\
\bar{L}_{32} & =L_{32}\left(Z_{22}^{*} L_{22}\right)^{-1} \bar{L}_{22}
\end{aligned}\right.
$$

Note that the transformation of $\hat{a}_{i}$ and $\hat{a}_{i+1}$ with $Z_{22}{ }^{*}$, only has an effect on the $i$ th and $(i+1)$ th row and column of $L$ and $D$.
Hence, the recovery of the triangular decomposition after the two-dimensional transformation has been applied, is rather straightforward. This is also understandable, considering the properties of sequential conditional least-squares estimation. The two two-dimensional matrices $\bar{L}_{22}$ and $\bar{D}_{22}$ follow from the unique triangular decomposition of $Z_{22}^{*}\left(L_{22} D_{22} L_{22}^{*}\right) Z_{22}$. Matrix $\bar{L}_{22}$ is lower triangular, having ones on its diagonal and $\sigma_{z(i+1, i \mid l)} \sigma_{\tilde{z}(i|, i,|)}^{-1}$ as its off-diagonal entry. This off-diagonal entry is in absolute value less than or equal to $1 / 2$. Compare with the triangular factor of (45). The diagonal matrix $\bar{D}_{22}$ has the conditional variances $\sigma_{\hat{z}(i|l, i| n)}$ and $\sigma_{\hat{z}(i+1|I+1, i+1| I+1)}$ as its entries and they satisfy the inequality (46).

The above result implies for $i=3$, that we are able to close the large gap between the third and fourth conditional variance in the spectrum. But of course, after the transformation has been applied, other, but smaller discontinuities emerge. For instance, if the transformation has been applied for $i=3$, then $\sigma_{\hat{z}(3|2,1 ; 3| 2,1)}<\sigma_{\hat{a}(2|1,2| 1)}$ and $\sigma_{\hat{a}(5|4| \ldots, 1 ; 5 \mid 4 \ldots, 1)}<\sigma_{\tilde{z}(4|3,2,1 ; 4| 3,2,1)}$. But also they can be removed by applying the two-dimensional transformation. The idea is therefore to continue applying the transformation to pairs of neighbouring ambiguities until the complete spectrum of conditional variances is flattened and (46) holds true for all $i$. Once this has been completed, the $n$-dimensional ambiguity transformation $Z^{*}$ is known and the original least-squares ambiguity vector $\hat{a}$ can be transformed as $\hat{z}=Z^{*} \hat{a}$. Its variance-covariance matrix is given as $Q_{\hat{z}}=Z^{*} Q_{\hat{a}} Z$ and the entries of the diagonal matrix $\bar{D}$ of its triangular decomposition $Q_{\bar{z}}=\bar{L} \bar{D} \bar{L}^{*}$ will then contain the transformed spectrum of conditional variances. The following example illustrates how the ambiguity transformation $Z^{*}$ succeeds in flattening the spectrum of conditional variances.

\section{Example 4}

This example is based on the same data as example 2. Figure 4 shows both the conditional standard deviations of the original least-squares ambiguities $\hat{a}_{i}$, as well as the conditional standard deviations of the transformed leastsquares ambiguities $\hat{z}_{i}$. We clearly observe a dramatic improvement in the spectrum. The discontinuity has been removed and the transformed conditional standard deviations are all of about the same small order. The dramatic decrease in value of the first three conditional standard deviations and their levelling with the remaining conditional standard deviations, now implies that our search for the integer leastsquares ambiguities already commences with very tight bounds, thus assuring with a very high likelihood that the first chosen integer candidates are indeed the coordinates of 
the sought for integer least-squares ambiguity vector $\check{z}$.

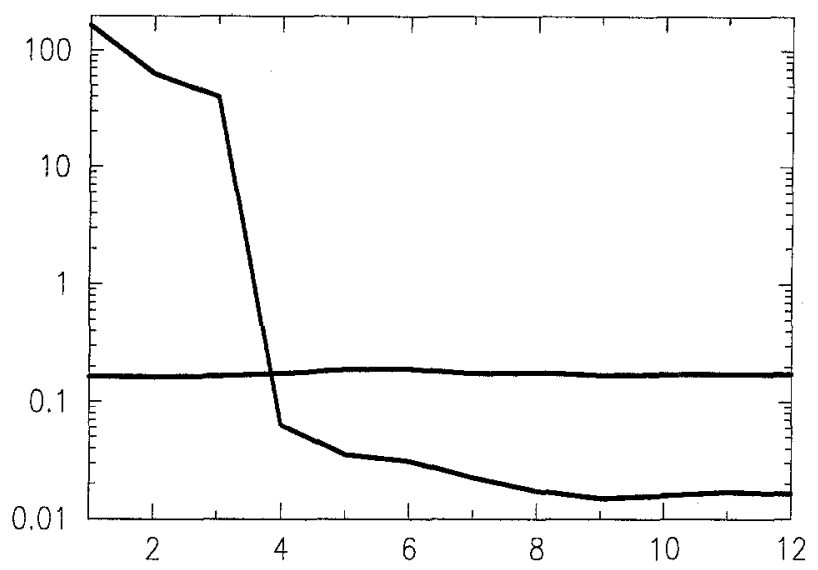

Figure 4: The original and transformed spectrum of conditional standard deviations.

Together with a bounding of the triangular factor as discussed above, the very low level of the transformed spectrum also allows us to assure that all unconditional variances of the transformed ambiguities are of the same small order.

To conclude this chapter, the following section will show how the spectrum of conditional variances can also be used to determine the gain in baseline precision that is achieved through the fixing of the ambiguities.

\subsection{On the Spectrum and the Gain in Baseline Precision.}

As it was remarked in section 2.2, the purpose of ambiguity fixing is to improve the precision of the baseline solution. This suggests that the precision of the least-squares ambiguities can be seen as an indicator of the amount of improvement one can achieve in the baseline precision. This indicator-property will be proven by showing the relation that exists between the determinants of $Q_{\hat{a}}, Q_{\hat{b}}$ and $Q_{\hat{b} \mid a}$.

For the conditional variance-covariance matrices $Q_{\hat{b} \mid a}$ and $Q_{\hat{a} \mid b}$ we may write: $Q_{\hat{b} \mid a}=\left(I-Q_{\hat{b} \hat{a}} Q_{\hat{a}}^{-1} \cdot Q_{\hat{a} \hat{b}} Q_{\hat{b}}^{-1}\right) Q_{\hat{b}}$ and $Q_{\hat{\alpha} \mid b}=\left(I-Q_{\hat{a} \hat{b}} Q_{\hat{b}}^{-1} \cdot Q_{\hat{b} \hat{a}} Q_{\hat{a}}^{-1}\right) Q_{\hat{a}}$. From this follows, together with the determinant identity $\operatorname{det}\left(I-Q_{\hat{b} \hat{a}} Q_{\hat{a}}^{-1}, Q_{\hat{a} b} Q_{\hat{b}}^{-1}\right)=$ $\operatorname{det}\left(I-Q_{\hat{a} \hat{b}} Q_{\hat{b}}^{-1} \cdot Q_{\hat{b} \hat{a}} Q_{\hat{a}}^{-1}\right)$, that

$$
\frac{\operatorname{det}\left(Q_{\hat{b}}\right)}{\operatorname{det}\left(Q_{\hat{b} \mid a}\right)}=\frac{\operatorname{det}\left(Q_{\hat{a}}\right)}{\operatorname{det}\left(Q_{\hat{a} \mid b}\right)}
$$

This relation is generally valid and it is independent of whether one is dealing with a single baseline model or a model where several baselines are adjusted for simultaneously. Note that $\operatorname{det}\left(Q_{\hat{a}}\right)$ equals the product of all ambiguity conditional variances $\sigma_{a(i|I, i| \Omega)}$ for $i=1, \ldots, n$. Also note that one may replace the dependence on the original ambiguities in (50) by the transformed ambiguities.

The variance-covariance matrix $Q_{\hat{a} \mid b}$ in (50) describes the precision of the least-squares double-difference ambiguities conditioned on a fixing of the baseline(s). Hence, $Q_{\hat{a} \mid b}$ does not depend on the relative receiver-satellite geometry. Its determinant is therefore easily determined. For a single baseline model, where an $s$-number of satellites are tracked over a $k$-number of epochs, we have for the single-frequency and dual-frequency case

$$
\left\{\begin{aligned}
L_{1}: \operatorname{det}\left(Q_{\hat{a} \mid b}\right) & =s\left(\sigma_{1}^{2} / k \lambda_{1}^{2}\right)^{s-1} \\
L_{1}, L_{2}: \operatorname{det}\left(Q_{\hat{a} \mid b}\right) & =s\left(\sigma_{1}^{2} / k \lambda_{1}^{2}\right)^{s-1} s\left(\sigma_{2}^{2} / k \lambda_{2}^{2}\right)^{s-1},
\end{aligned}\right.
$$

in which $\sigma_{1}^{2}$ and $\sigma_{2}^{2}$ are the a-priori variances of the singledifferenced carrier-phase observables on $L_{1}$ and $L_{2}$. This result can now be used in combination with (50) to show how the gain in baseline precision is governed by the spectrum of conditional variances. For instance, for the single-frequency single baseline case we get

$$
\frac{\operatorname{det}\left(Q_{\hat{b}}\right)}{\operatorname{det}\left(Q_{\hat{b} \mid a}\right)}=\left[\prod_{i=1}^{n}\left(k \lambda_{1}^{2} \sigma_{\hat{z}(i|I, i| n)} / \sigma_{1}^{2}\right)\right] /(n+1) .
$$

Note that the square-root of the left-hand side of this expression equals the volume of the three-dimensional standard ellipsoid of the "floated" baseline, divided by the volume of the three-dimensional standard ellipsoid of the "fixed" baseline. Also note, since the left-hand side of (52) is independent of the chosen reference satellite in the doubledifference ambiguities, that also the right-hand side must be independent of this choice. Hence, although the individual conditional variances are dependent on the choice of reference satellite, their product is not.

\section{Summary and Concluding Remarks}

In this contribution a method for the fast estimation of the integer least-squares ambiguities has been introduced. The basic idea that lies at the root of the method is that integer ambiguity estimation becomes trivial once the ambiguities are decorrelated. The approach followed is therefore to aim at decorrelating the least-squares ambiguities, while retaining their intrinsic integer nature. The method consists of two steps, a transformation step and a search step, both of which rely on the results of a sequential conditional least-squares ambiguity adjustment. The transformation-step starts from extending current usage of single-channel integer ambiguity 
linear combinations to invertible multi-channel linear combinations. The importance of this extension is, that it provides significant leeway to influence the dependence of the ambiguity variance-covariance matrix on the designmatrix containing the receiver-satellite geometry. For short observational timespans, this dependence has been identified as a discontinuity in the spectrum of ambiguity conditional variances. As a consequence a direct search for the original integer least-squares ambiguities will be rather timeconsuming. Based on an integer approximation of a fully decorrelating transformation, transformed conditional leastsquares ambiguities were recovered having an almost flattened spectrum. As a result, the search for the transformed integer least-squares ambiguities already commences with tight bounds, thus assuring that the solution can be found in a highly efficient manner.

As it was stressed in the introduction, our method is concerned with the estimation problem and not with the validation problem. Hence, a proper validation should still be used after the integer least-squares ambiguities have been identified. And since the quadratic forms which are usually used for validation purposes are invariant to our reparametrization (e.g. $\|\hat{a}-\breve{a}\|_{Q_{a}}^{2}=\left\|\hat{z}-z_{z}\right\|_{Q_{\varepsilon}}^{2}$ ), any of the existing validation procedures can be used. Also note, that one can use, if so desired, ones own search procedure. But instead of applying it to the original ambiguities, it should then be applied to our transformed ambiguities.

We would also like to remark that the ambiguity transformation used, is completely determined by the variance-covariance matrix of the ambiguities. Even the a posteriori variance-factor need not be known. This stipulates that actual measurements are not needed for the transformation-step. Hence, the necessary computations for this step can be done in principle at the designing stage, prior to the actual measurement stage.

In sections 2.1 and 2.2 we have followed the customary practice of working with the double-difference version of the carrier-phase observation equations. This however, is not really necessary. One could as well work with the undifferenced version of the carrier-phase observation equations, as long as the original set of undifferenced noninteger ambiguities is reparametrized into a set of integer ambiguities plus a remaining set of noninteger parameters. In fact, this is precisely the approach used in our numerical software implementation. Similar approaches have been advocated in [13] and [30]. In sections 2.1 and 2.2 we have, for reasons of simplicity, also restricted ourselves to a parametrization in which only the ambiguities and baseline components appear as parameters. But if needed and when estimable, additional parameters (e.g. different types of delays) can be included without affecting the principle of the approach. On the observational side, the method is also independent in principle, on whether code data are used or not, and on whether dual-frequency data are used or not. And when dual-frequency data are used, one could also apply the method to other type of ambiguities, such as the wide-lane or narrow-lane ambiguities.

\section{Acknowledgement}

The author acknowledges the assistance of ir. P.J. de Jonge and ir. C.C.J.M. Tiberius for preparing the numerical examples.

\section{References}

[1] Hatch, R. (1986): Dynamic Differential GPS at the Centimeter Level. Proceedings 4th International Geod. Symp. on Satellite Positioning, Austin, Texas, 28 April 2 May, pp. 1287-1298.

[2] Hofmann-Wellenhof, B., B.W. Remondi (1988): The Antenna Exchange: one Aspect of High-Precision Kinematic Survey. Presented at the International GPS Workshop, GPS Techniques Applied to Geodesy and Surveying, Darmstadt, FRG, 10-13 April.

[3] Remondi, B.W. (1984): Using the Global Positioning System (GPS) Phase Observable for Relative Geodesy: Modelling, Processing, and Results, Ph.D. dissertation, NOAA, Rockville, 360 pp..

[4] Seeber, G. G. Wübbena (1989): Kinematic Positioning with Carrier Phases and "On the Way" Ambiguity Solution. Proceedings 5th Int. Geod. Symp. on Satellite Positioning. Las Cruces, New Mexico, March 1989.

[5] Frei, E., G. Beutler (1990): Rapid Static Positioning Based on the Fast Ambiguity Resolution Approach FARA: Theory and First Results. Manuscripta Geodaetica, Vol. 15, No. 6, 1990.

[6] Blewitt, G.(1990): A New Tool for Dense Monitoring of Crustal Strain: GPS Rapid Static Surveying. EOS Transaction American Geophysical Union, Vol. 71, No. 17, p. 483.

[7] Euler, H.-J., C. Goad (1990): On Optimal Filtering of GPS Dual Frequency Observations without using Orbit Information. Bulletin Geodesique, Vol. 65, pp. 130-143.

[8] Kleusberg, A. (1990): A Review of Kinematic and 
Static GPS Surveying Procedures. Proceedings of the Second International Symposium on Precise Positioning with the global Positioning system, Ottawa, Canada, September 3-7 1990, pp. 1102-1113.

[9] Counselman, C.C., S.A. Gourevitch (1981): Miniature Interferometer Terminals for Earth Surveying: Ambiguity and Multipath with Global Positioning System. IEEE Transactions on Geoscience and Remote Sensing, Vol. GE-19, No. 4, pp. 244-252.

[10] Hatch, R. (1989): Ambiguity Resolution in the Fast Lane. Proceedings ION GPS-89, Colorado Springs, CO, 27-29 September, pp. 45-50.

[11] Remondi, B.W. (1986): Performing Centimeter-Level Surveys in Seconds with GPS Carrier Phase: Initial Results. Journal of Navigation, Volume III, The Institute of Navigation.

[12] Remondi, B.W. (1991): Pseudo-Kinematic GPS Results Using the Ambiguity Function Method, Journal of Navigation, Vol. 38, No. 1, pp. 17-36.

[13] Blewitt, G. (1989): Carrier Phase Ambiguity Resolution for the Global Positioning System Applied to Geodetic Baselines up to $2000 \mathrm{~km}$. Journal of Geophysical Research, Vol. 94, No. B8, pp. 10.187-10.203.

[14] Hatch, R. (1991): Instantaneous Ambiguity Resolution. Proceedings of IAG International Symposium 107 on Kinematic Systems in Geodesy, Surveying and Remote Sensing, Sept. 10-13, 1990, Springer Verlag, New York, pp. 299-308.

[15] Frei, E. (1991): Rapid Differential Positioning with the Global Positioning System. In: Geodetic and Geophysical Studies in Switzerland, Vol. 44.

[16] Wübbena, G. (1991): Zur Modellierung von GPSBeobachtungen fur die hochgenaue Positionsbestimmung, Hannover, 1991.

[17] Euler, H.-J., H. Landau (1992): Fast GPS Ambiguity Resolution On-The-Fly for Real-Time Applications. Proceedings 6th Int. Geod. Symp. on Satellite Positioning. Columbus, Ohio, 17-20 March 1992, pp. 650-729.

[18] Mervart, L., G. Beutler, M. Rothacher, U. Wild (1994): Ambiguity Resolution Strategies using the Results of the International GPS Geodynamics Service (IGS), Bulletin Geodesique, 68: 29-38.

[19] Teunissen, P.J.G. (1993): The Invertible GPS Ambiguity Transformations. Delft Geodetic Computing Centre (LGR), $9 \mathrm{p}$.

[20] Wübbena, G. (1989): The GPS Adjustment Software Package - GEONAP - Concepts and Models. Proceedings 5th Int. Geod. Symp. on Satellite Positioning. Las Cruces, New Mexico, 13-17 March
1989, pp. 452-461.

[21] Allison, T. (1991): Multi-Observable Processing Techniques for Precise Relative Positioning. Proceedings ION GPS-91. Albuquerque, New Mexico, 11-13 September 1991, pp. 715-725.

[22] Goad, C. (1992) Robust Techniques for Determining GPS Phase Ambiguities. Proceedings 6th Int. Geod. Symp. on Satellite Positioning. Columbus, Ohio, 17-20 March 1992, pp. 245-254.

[23] Cocard, C., A. Geiger (1992): Systematic Search for all Possible Widelanes. Proceedings 6th Int. Geod. Symp. on Satellite Positioning. Columbus, Ohio, 17-20 March 1992.

[24] Teunissen, P.J.G. (1993): Least-Squares Estimation of the Integer GPS Ambiguities. Delft Geodetic Computing Centre (LGR), 16 p. Invited Lecture, Section IV Theory and Methodology. IAG General Meeting, Beijing, China, August 1993. Also in LGR-Series no. 6.

[25] Golub, G.H. and C.F. Van Loan (1986): Matrix Computations. North Oxford Academic.

[26] Lenstra, H.W. (1981): Integer Programming with a Fixed Number of Variables. University of Amsterdam, Dept. of Mathematics, report 81-03.

[27] Teunissen, P.J.G. (1990): GPS op afstand bekeken (in Dutch). In: Een halve eeuw in de goede richting. Lustrumboek Snellius 1950-1990, pp. 215-233.

[28] Euler, H.-J., B. Schaffrin (1991): On a Measure for the Discernability between Different Ambiguity Resolutions in the Static-Kinematic GPS-mode. Proceedings of $I A G$ International Symposium 107 on Kinematic Systems in Geodesy, Surveying and Remote Sensing, Sept. 10-13, 1990, Springer Verlag, New York, pp. 285-295.

[29] Betti, B., M. Crespi, and F. Sanso (1993): A Geometric Illustration of Ambiguity Resolution in GPS Theory and a Bayesian Approach, Manuscripta Geodaetica 18: 317-330.

[30] Goad, C.C. (1985): Precise Relative Position Determination Using Global Positioning System Carrier Phase Measurements in a Nondifference Mode. Proceedings of First Int. Symposium on Precise Positioning with the Global Positioning System, April 15-19, 1985, Rockville, Maryland, Vol. 1, pp. 347-356, U.S. Department of Commerce, National Geodetic Information Center, NOAA. 